\title{
Strong interaction effects at a Fermi surface in a model for voltage-biased bilayer graphene
}

\author{
Wes Armour, ${ }^{1}$ Simon Hands, ${ }^{2}$ and Costas Strouthos ${ }^{3}$ \\ ${ }^{1}$ Oxford e-Research Centre, University of Oxford, 7 Keble Road, Oxford OX1 3QG, United Kingdom \\ ${ }^{2}$ Department of Physics, College of Science, Swansea University, Singleton Park, Swansea SA2 8PP, United Kingdom \\ ${ }^{3}$ School of Sciences, Department of Computer Science, European University Cyprus, 1516 Cyprus \\ (Received 18 September 2015; revised manuscript received 24 November 2015; published 23 December 2015)
}

\begin{abstract}
Monte Carlo simulation of a $2+1$ dimensional model of voltage-biased bilayer graphene, consisting of relativistic fermions with chemical potential $\mu$ coupled to charged excitations with opposite sign on each layer, has exposed noncanonical scaling of bulk observables near a quantum critical point found at strong coupling. We present a calculation of the quasiparticle dispersion relation $E(k)$ as a function of exciton source $j$ in the same system, employing partially twisted boundary conditions to boost the number of available momentum modes. The Fermi momentum $k_{F}$ and superfluid gap $\Delta$ are extracted in the $j \rightarrow 0$ limit for three different values of $\mu$, and support a strongly interacting scenario at the Fermi surface with $\Delta \sim O(\mu)$. We propose an explanation for the observation $\mu<k_{F}$ in terms of a dynamical critical exponent $z<1$.
\end{abstract}

DOI: 10.1103/PhysRevB.92.235143

PACS number(s): 11.10.Kk, 11.15.Ha, 73.63.Bd, 73.22.Pr

\section{INTRODUCTION}

There are very few many-body systems permitting Monte Carlo simulation without the need to confront a sign problem. In Ref. [1] we introduced a new member to this class, based on an effective theory of bilayer graphene in which charge carrying excitations are modeled as $N_{f}=4$ relativistic fermions moving in a $2 d$ plane. The introduction of chemical potential $\mu$ is via a bias voltage in the perpendicular direction which induces equal densities of electrons on one layer and holes on the other; this is analogous to isospin chemical potential in QCD and yields a real positive fermion determinant amenable to orthodox Monte Carlo methods. The simulation was performed in the vicinity of a quantum critical point (QCP) found at strong coupling, and the main result was the demonstration that the ground state is a superfluid formed by condensation of electron-hole exciton pairs, and that the response to $\mu$ is governed by the noncanonical scaling forms (8) and (9) given in Sec. II. We expect the exciton condensation to result in an energy gap $\Delta>0$ developing at the Dirac points.

The model [1] as originally devised for bilayer graphene [2] is artificial in a few respects. First, the description in terms of $N_{f}=4$ relativistic species (i.e., $N_{f}=2$ electrons and $N_{f}=2$ holes, where $N_{f}$ counts the number of fourcomponent spinors) is only justified by the band structure of the tight-binding model in the presence of an interlayer "skew" coupling breaking the trigonal symmetry of the underlying lattice [3]. Second, the interaction between charge densities is simplified to be a local four-Fermi contact, although a more realistic unscreened Coulomb interaction can be modeled with the introduction of a third spatial lattice direction to capture the electrodynamics [4]. Finally, intra- and interlayer interactions have the same coupling strength, as a necessary condition of keeping the fermion determinant real. Nonetheless, it shares

Published by the American Physical Society under the terms of the Creative Commons Attribution 3.0 License. Further distribution of this work must maintain attribution to the author(s) and the published article's title, journal citation, and DOI. the essential features of a more general model for doublelayer graphene systems in which there is some hybridization permitting interlayer tunneling [5]. With $N_{f}=1$ this approach is also applicable to surface states of topological insulators, motivating study with variable $N_{f}$ [6].

Within the literature there are discrepant predictions for the strength of the gap $\Delta$ generated by exciton condensation in biased bilayer systems. Kharitonov and Efetov [7] treat a Coulomb interaction among $N_{f}=4$ flavors (in our normalization) using a large- $N_{f}$ approach (equivalent to the random phase approximation) and treat the polarization operator $\Pi(\omega, q)$ due to electron-hole pairs in the static limit $\omega \rightarrow 0$, in effect using its value in the normal state. They find a very small value $\Delta / \mu \sim e^{-4 N_{f}} \sim O\left(10^{-7}\right)$. Sodemann et al. [5] take the $\omega$ dependence of $\Pi$ into account, thereby modeling the retarded character of the polarization induced by relativistic degrees of freedom, and find a significant reduction of screening as $q, \omega \rightarrow 0$ in a gapped phase with $\Delta>0$. They find $\Delta / \mu \sim O(1)$ for values of the effective fine structure constant $\alpha=e^{2} / \varepsilon v_{F}>\alpha_{c} \sim 1$.5. In the current paper, although the classical potential is screened $a b$ initio, quantum screening effects are treated consistently using relativistic field theory; the distinctive feature is that results are obtained in the vicinity of a QCP corresponding to a strong coupling intralayer transition to a Mott insulating phase, known to exist at $\mu=0$ from Monte Carlo simulations [6].

The results of [1] were interpreted in terms of strong interaction effects at a Fermi surface; if exciton pairs within a shell of thickness $\Delta$ condense around the Fermi surface centered at $k_{F}$, then the anomalous scaling $(8,9)$ is consistent with a BCS mechanism with $\Delta \sim O(\mu)$. Everything is to be viewed in the context of an effective field theory valid near the QCP. In order to put this picture on firmer footing, and also to expose the Fermi surface, in this paper we use Monte Carlo simulation to calculate the quasiparticle dispersion relation $E(k)$, identifying $k_{F}$ with the location of the minimum and $\Delta$ with $E\left(k_{F}\right)$. The main results are summarized in Fig. 5 below. In Sec. II we present the model and review the main findings of [1], then in Sec. III present the calculation of $E(k)$. Our results, summarized in Sec. IV, indeed support the picture of a Fermi surface disrupted by strong interactions, leading to the 
formation of a gap $\Delta$ increasing monotonically with $\mu$. We also discuss our observation of the striking inequality $\mu<k_{F}$, and propose an explanation in terms of an estimate for the dynamical critical exponent $z<1$.

\section{FORMULATION AND SIMULATION OF THE MODEL}

Here we recap the formulation of a model for low-energy electron excitations in bilayer graphene introduced in [1]. The main physical idea is based on $N_{f}=2$ flavors of relativistic fermion moving within each layer, described by Grassmann fields $\psi, \bar{\psi}$, with (Euclidean) action $[8,9]$ :

$$
\begin{aligned}
S_{\mathrm{mono}}= & \sum_{a=1,2} \int d x_{0} d^{2} x\left(\bar{\psi}_{a} \gamma_{0} \partial_{0} \psi_{a}+v_{F} \bar{\psi}_{a} \vec{\gamma} \cdot \vec{\nabla} \psi_{a}\right. \\
& \left.+i A_{0} \bar{\psi}_{a} \gamma_{0} \psi_{a}\right)+\frac{1}{2 e^{2}} \int d x_{0} d^{3} x\left(\partial_{i} A_{0}\right)^{2} .
\end{aligned}
$$

Here $e$ is the effective electron charge whose value depends on the dielectric properties of the substrate, the $4 \times 4$ Dirac matrices satisfy $\left\{\gamma_{\mu}, \gamma_{\nu}\right\}=2 \delta_{\mu \nu}, \mu=0,1,2$, and $A_{0}$ is a fluctuating $3+1 d$ electrostatic potential field sourced by the charge density $\bar{\psi} \gamma_{0} \psi ; A_{0}$ is a remnant of the full electromagnetic field in the instantaneous approximation justified for Fermi velocity $v_{F} \ll c$.

A description of bilayer graphene in terms of $N_{f}=4$ relativistic fermions requires some justification. For a perfect bilayer formed from two monolayers stacked in $\mathrm{AB}$ configuration with interlayer coupling strength $t^{\prime} \sim O(0.1) t$, where $t$ is the hopping parameter in the original tight-binding Hamiltonian, the electron dispersion relation in the vicinity of the Dirac point is quadratic, only becoming approximately relativistic (i.e., linear) for $k a \gtrsim t^{\prime} / t$ [10]. Theoretical studies suggest however that the presence of a "skew" interlayer coupling with strength $t_{3}$ breaking the trigonal symmetry of the crystal, the parabolic bands split to form separate Dirac cones so that $N_{f}=4$ is a reasonable approximation for $k a \lesssim t_{3} t^{\prime} / t^{2}$ [3]. A similar effect results from mechanical deformation.

The second ingredient is that the layers are given equal and opposite constant bias voltages $\pm \mu$, inducing on one layer a negatively charged concentration of particles and on the other a positively charged concentration of holes. As the notation implies, the bias voltage is precisely a chemical potential, in this case formally similar to an isospin chemical potential in QCD. Euclidean formulations of systems with $\mu \neq 0$ are generically afflicted with a "sign problem," i.e., the Lagrangian density $\mathcal{L}$ is no longer positive definite, or even real, since the inequivalence under time reversal translates into inequivalence under complex conjugation in Euclidean metric. However, the case of isospin chemical potential is known not to have a sign problem and is hence simulable using orthodox Monte Carlo methods.

The model we use to describe voltage-biased bilayer graphene in terms of $N_{f}=4$ relativistic fermions is described by the following Lagrangian [1]:

$$
\begin{aligned}
\mathcal{L} & =(\bar{\psi}, \bar{\phi})\left(\begin{array}{cc}
D[V ; \mu] & i j \\
-i j & D[V ;-\mu]
\end{array}\right)\left(\begin{array}{l}
\psi \\
\phi
\end{array}\right)+\frac{1}{2 g^{2}} V^{2} \\
& \equiv \bar{\Psi} \mathcal{M} \Psi+\frac{1}{2 g^{2}} V^{2} .
\end{aligned}
$$

Here $\psi$ and $\phi$ are $4 \times 2$-component spinors each describing two Dirac flavors, with $\psi, \bar{\psi}$ corresponding to electron degrees of freedom on one layer and $\phi, \bar{\phi}$ holes on the other; $V$ is an auxiliary field defined on the timelike links of the lattice yielding a screened version of the electrostatic potential in (1); and $j$ a symmetry-breaking gap parameter due to interlayer pairing. Although $V$ defined in this way yields a potential with no classical Coulomb $r^{-1}$ tail, it was shown in [6] that $V$ and $A_{0}$ in (1) have the same large- $N_{f}$ quantum corrections, and the models coincide in the strong coupling limit $e^{2}, g^{2} \rightarrow$ $\infty$. Because some weak interlayer hybridization is likely to be present in double-layer systems, in general $j \neq 0$ [5]; however we will attempt to extrapolate $j \rightarrow 0$ so that exciton condensation can be viewed as a spontaneous symmetry breaking $U(4) \otimes U(4) \rightarrow U(4)$ [1]. In continuum notation the covariant derivative operator including the bias voltage is

$$
\begin{aligned}
D[V ; \mu] & =\delta^{\alpha, \beta}\left(\sum_{\nu=0, \ldots, 2} \gamma_{\nu} \partial_{\nu}+(i V+\mu) \gamma_{0}\right) \\
& =-D^{\dagger}[V ;-\mu],
\end{aligned}
$$

where $\alpha, \beta$ run over $N_{f}=2$ Dirac flavors. The minimal coupling to $V$ implies that $\psi \psi, \phi \phi$, and $\phi \psi$ interactions are all of equal strength, which is required for the action to be real following integration over the fermions. This corresponds to the interlayer separation $d \rightarrow 0$ in the double-layer model [5].

In terms of staggered fermion fields living on the sites $x, y$ of a $2+1 d$ cubic lattice $D$ is written

$$
\begin{aligned}
D_{x, y}^{\text {latt }}= & \frac{1}{2}\left[\eta_{0 x} e^{\mu}\left(1+i V_{x}\right) \delta_{y, x+\hat{0}}-\eta_{0 x} e^{-\mu}\left(1-i V_{x}\right) \delta_{y, x-\hat{0}}\right. \\
& \left.+\sum_{\nu=1,2} \eta_{\nu x}\left(\delta_{y, x+\hat{v}}-\delta_{y, x-\hat{v}}\right)\right]
\end{aligned}
$$

where the sign factors $\eta_{v x} \equiv(-1)^{x_{0}+\cdots+x_{v-1}}$ ensure a covariant weak-coupling continuum limit. Equation (4) was also used to model electron excitations in monolayer graphene [11]. Note that in $2+1 d$ a single staggered fermion automatically describes $N_{f}=2$ continuum flavors [12]. We work in units in which both spatial $a_{s}$ and temporal $a_{t}$ lattice spacings are set to unity (equivalent to setting the bare Fermi velocity $v_{F}=1$ ); note that for a noncovariant action there is no reason a priori to assume $a_{s}=a_{t}$, though since the value of the ratio $a_{t} / a_{s}$ is driven by UV physics it does not depend on $\mu$ and only weakly on $j$, so may be assumed constant throughout this paper. On the assumption that the dimension of $D^{\text {latt }}$ is even, it is straightforward to use (3) to show

$$
\operatorname{det} \mathcal{M}=\operatorname{det}\left[D^{\dagger} D+j^{2}\right]>0
$$

and hence there is no obstruction to Monte Carlo simulation using orthodox numerical techniques.

In [1] we presented results from numerical simulation of the model (2) and (4) using a hybrid Monte Carlo algorithm. The coupling $g^{-2} a^{\prime}=0.4$, where the factor $a^{\prime}=a_{s}^{2} a_{t}^{-1}$ follows because the interaction couples charge densities, was chosen in the vicinity of the quantum critical point (QCP), although since $N_{f}=4 \lesssim N_{f c}=4.8(2)$ [6] it is hard to ascertain with confidence on which side of the phase boundary at $\mu=0$ we 
are sitting. Two principal observables were monitored as a function of $\mu$ and $j$, namely the carrier density

$$
n_{c} \equiv \frac{1}{2} \frac{\partial \ln \mathcal{Z}}{\partial \mu}=\frac{1}{2}\left\langle\bar{\psi} D_{0} \psi-\bar{\phi} D_{0} \phi\right\rangle,
$$

and the exciton condensate

$$
\langle\Psi \Psi\rangle \equiv \frac{\partial \ln \mathcal{Z}}{\partial j}=i\langle\bar{\psi} \phi-\bar{\phi} \psi\rangle .
$$

Following extrapolation to the limit $j \rightarrow 0$ (so long as $\mu a_{t}<0.3$ at which point saturation artifacts set in and the continuum approximation fails), both observables showed behavior consistent with rising smoothly from zero as $\mu$ is increased, with

$$
\begin{gathered}
n_{c} \propto \mu^{3.32(1)}, \\
\langle\Psi \Psi\rangle \propto \mu^{2.39(2)} .
\end{gathered}
$$

This is to be contrasted with the expectations from weak coupling. In free field theory the carrier density depends on the volume contained within the Fermi surface; for relativistic fermions $k_{F} \approx \mu$ and hence in $2+1 d n_{c} \propto \mu^{2}$. Similarly, in weak coupling we expect the exciton condensate to arise from electron-hole pairing with equal and opposite momenta from within a shell of thickness $2 \Delta$ centered on $k_{F}$; hence $\langle\Psi \Psi\rangle \propto$ $\Delta \mu$. The noncanonical scaling is taken to be a symptom of strong field fluctuations near the QCP. Moreover, since according to Luttinger's theorem $n_{c} \propto k_{F}^{2}$ even in the presence of interactions, we can adapt this argument to estimate the scaling of the gap $\Delta(\mu)$ :

$$
\Delta(\mu)=\frac{\langle\Psi \Psi(\mu)\rangle}{n_{c}^{\frac{1}{2}}(\mu)} \propto \begin{cases}\text { constant } & \text { weak coupling } \\ \mu^{1.44(1)} & \text { near QCP. }\end{cases}
$$

While the numerical value for the exponent should probably not be taken too seriously, the qualitative difference between the scaling predicted in weak coupling and observed near the QCP is striking; indeed, Fig. 13 of [1] shows the ratio $\langle\Psi \Psi\rangle / n_{c}^{1 / 2}$ extrapolated to $j \rightarrow 0$ almost linearly proportional to $\mu$. Since near a QCP $\mu$ is the only energy scale in the problem, naively $\Delta \propto \mu$ is expected. As both arguments contain assumptions, it is clear that a direct calculation of $\Delta$ from the quasiparticle propagator $\langle\Psi(0) \bar{\Psi}(x)\rangle$ is needed.

\section{QUASIPARTICLE DISPERSION}

While the results outlined in the previous section are intriguing, the conclusions, drawn from simulations of systems with both significant UV and IR artifacts on a restricted range of $\mu$ values, are necessarily provisional. In this paper we will present complementary information through analysis of the quasiparticle dispersion relation $E(\vec{k})$, which will enable identification of the Fermi momentum $k_{F}$ and direct estimation of the superfluid gap $\Delta$. The basic observables are the timeslice correlators in momentum space:

$$
\begin{aligned}
& C_{N}(\vec{k}, t)=\sum_{\vec{x}}\langle\psi(\overrightarrow{0}, 0) \bar{\psi}(\vec{x}, t)\rangle e^{-i \vec{k} \cdot \vec{x}}, \\
& C_{A}(\vec{k}, t)=\sum_{\vec{x}}\langle\psi(\overrightarrow{0}, 0) \bar{\phi}(\vec{x}, t)\rangle e^{-i \vec{k} \cdot \vec{x}},
\end{aligned}
$$

where we distinguish between normal propagation of an electron or hole within a layer, and anomalous propagation in which an electron moving in one layer is absorbed by an exciton, transferring its momentum to an electron moving in the other. On a finite volume in the absence of explicit symmetry breaking $j=0$, the anomalous component $C_{A}$ necessarily vanishes.

Many momentum modes must be available in order to obtain a good resolution for $E(\vec{k})$. Naively this would entail making at least one of the spatial dimensions of the lattice as large as possible [13]. It is much more efficient, however, to employ partially twisted boundary conditions [14] in which the constraint $\psi\left(L_{x}\right)=e^{i \theta_{x}} \psi(0)$, with the angle $\theta_{x}$ adjustable, is implemented in the calculation of the propagator (11) so that accessible modes have

$$
k_{x}=\frac{2 \pi n+\theta_{x}}{L_{x}} .
$$

In practice the twist is implemented via a field redefinition so that each $x$ link in (4) is multiplied by a phase $e^{ \pm i \theta_{x} / L_{x}}$. Treating $+x$ and $-x$ independently, the choice $\theta_{x}=2 \pi / 3$ permits an effective tripling of the number of available modes for a given $L_{x}$ for the cost of an extra inversion (although equivalent statistics requires twice as many twisted inversions as nontwisted). For the two volumes investigated here, the accessible modes were $k a_{s}=0, \frac{\pi}{48}, \ldots, \frac{n \pi}{48}, \ldots,\left(32^{3}\right)$ and $k a_{s}=0, \frac{\pi}{72}, \ldots, \frac{n \pi}{72}, \ldots,\left(48^{3}\right)$. In all cases the maximum accessible momentum for staggered fermions is $\frac{\pi}{2}$.

Results were generated at $g^{-2} a^{\prime}=0.4$ at $\mu a_{t}=0.1,0.2$ on $32^{3}$ and $\mu a_{t}=0.1,0.15,0.2$ on $48^{3}$, with exciton source $j \tilde{a}=$ $0.005,0.01,0.02, \ldots, 0.05$ (here $\tilde{a}=a_{s}^{\alpha} a_{t}^{1-\alpha}$ with $\alpha$ defined by the renormalization prescription adopted for the nonconserved density $\bar{\psi} \phi)$. A hybrid Monte Carlo algorithm with $\delta \tau=$ 0.0025 and mean trajectory length $\bar{\tau}=2\left(32^{3}\right)$ or $\bar{\tau}=1\left(48^{3}\right)$ was used, and propagator measurements taken at the end of every trajectory using a randomly chosen point source. The results presented arise from $O(5000)$ measurements at $j \tilde{a}=$ 0.005 up to $O\left(3 \times 10^{4}\right)$ at $j \tilde{a}=0.05$. Since the conventional staggered fermion mass term is absent from (4), the global symmetry

$$
\begin{aligned}
\psi & \mapsto e^{i \alpha \varepsilon(x)} \psi, \quad \bar{\psi} \mapsto e^{i \alpha \varepsilon(x)} \bar{\psi}, \quad \phi \mapsto e^{-i \alpha \varepsilon(x)} \phi, \\
\bar{\phi} \mapsto e^{-i \alpha \varepsilon(x)} \bar{\phi}, &
\end{aligned}
$$

with $\varepsilon(x) \equiv(-1)^{x_{0}+x_{1}+x_{2}}$, means that $C_{N}(k, t)$ vanishes for $t$ even and $C_{A}(k, t)$ for $t$ odd. Moreover, only $\operatorname{Re} C_{N}$ and $\operatorname{Im} C_{A}$ survive the ensemble average. The resulting correlators are then fitted to the forms [15]

$$
\begin{aligned}
& C_{N}(k, t)=A e^{-E_{N} t}+B e^{-E_{N}\left(L_{t}-t\right)}, \\
& C_{A}(k, t)=C\left(e^{-E_{A} t}-e^{-E_{A}\left(L_{t}-t\right)}\right),
\end{aligned}
$$

to yield the $k$-dependent amplitudes $A, B,|C|$ and the energy $E$, which is extracted independently from both $C_{N}$ and $C_{A}$. Stable fits for $C_{N}(t)$ and $C_{A}(t-1)$ were found for the windows $t a_{t} \in[7,25]\left(32^{3}\right)$ and $t a_{t} \in[7,41]\left(48^{3}\right)$. It is slightly unusual to have to deal with excited state contamination when fitting a fermion propagator, which may be a symptom of the strong fluctuations near a QCP; this was found to be an issue particularly for $k \gtrsim k_{F}$. 


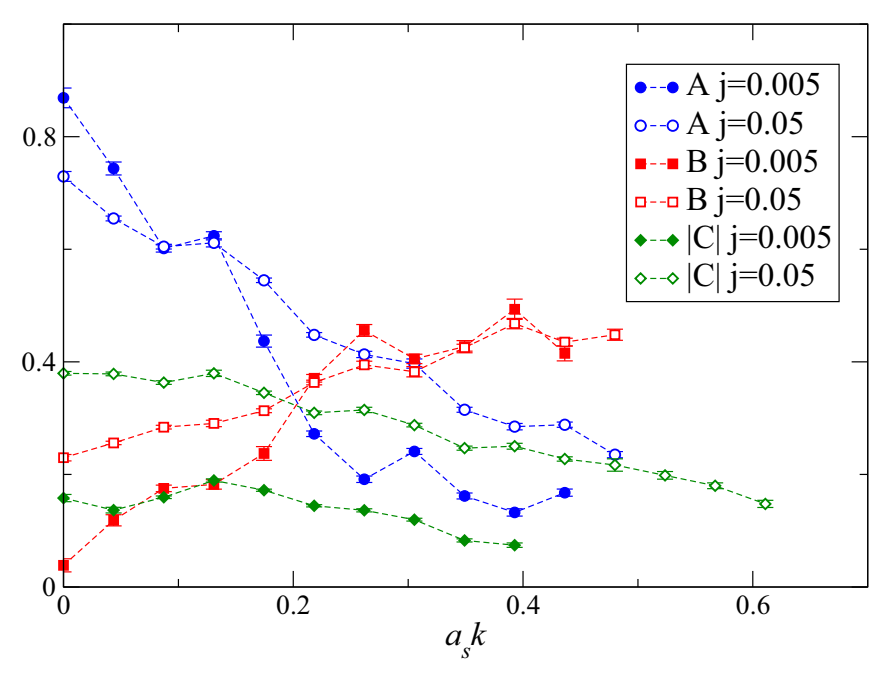

FIG. 1. (Color online) Amplitudes obtained from fits to (14) and (15) on $48^{3}$ at $\mu a_{t}=0.1$.

It is instructive first to consider the fitted amplitudes: Fig. 1 shows results from $\mu a_{t}=0.1$ on $48^{3}$, for two values of the exciton source $j$. In the normal channel the time-asymmetric form of $C_{N}$ is manifest, and changes in character as $k$ increases. For small $k$ the forwards propagating signal is stronger, but $B / A$ grows with $k$ and for $k a_{s} \gtrsim 0.2$ the backwards signal dominates. The interpretation is as follows [15]: for $k<k_{F}$ the dominant excitations are holelike, and for $k>k_{F}$ particlelike. Increasing $j \tilde{a}$ from 0.005 to 0.05 has the effect of smearing the Fermi surface so that quasiparticles tend to become an admixture of both, and the disparity between $A$ and $B$ diminishes. The effect of smearing is also seen in the anomalous channel, where $C_{A}$ grows steadily in magnitude with increasing $j$. In weakly coupled models $[13,15]|C(k)|$ is nonmonotonic with a maximum near $k_{F}$ where particle-hole mixing is strongest, but here the behavior is less clear cut. The same trends with both $k$ and $j$ are observed at larger $\mu$.

Figure 2 shows results from fits to the dispersion $E(k)$ for various $\mu$ at $j \tilde{a}=0.01$ from (14) and (15). The common

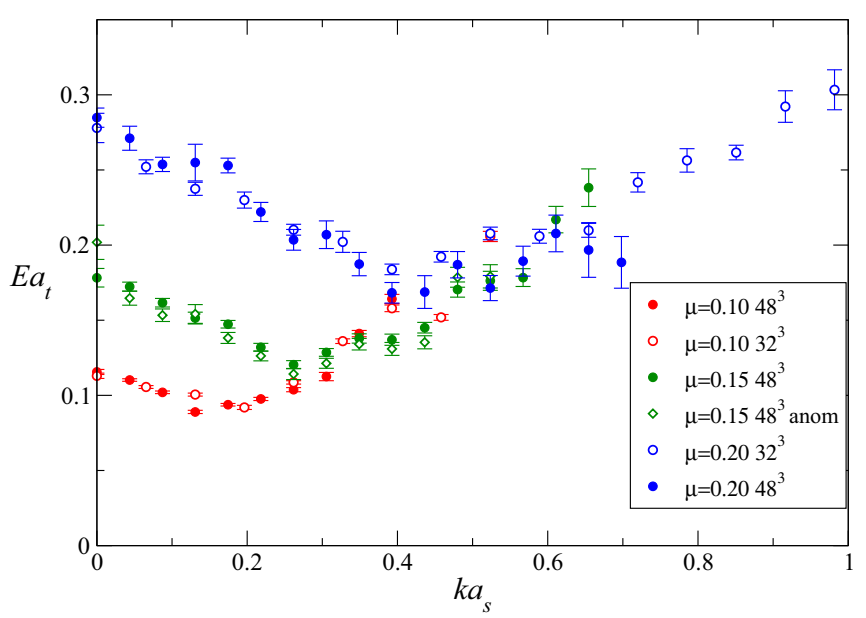

FIG. 2. (Color online) Dispersion relation $E(k)$ in the normal channel (unless stated) for various $\mu$, for fixed $j \tilde{a}=0.01$.

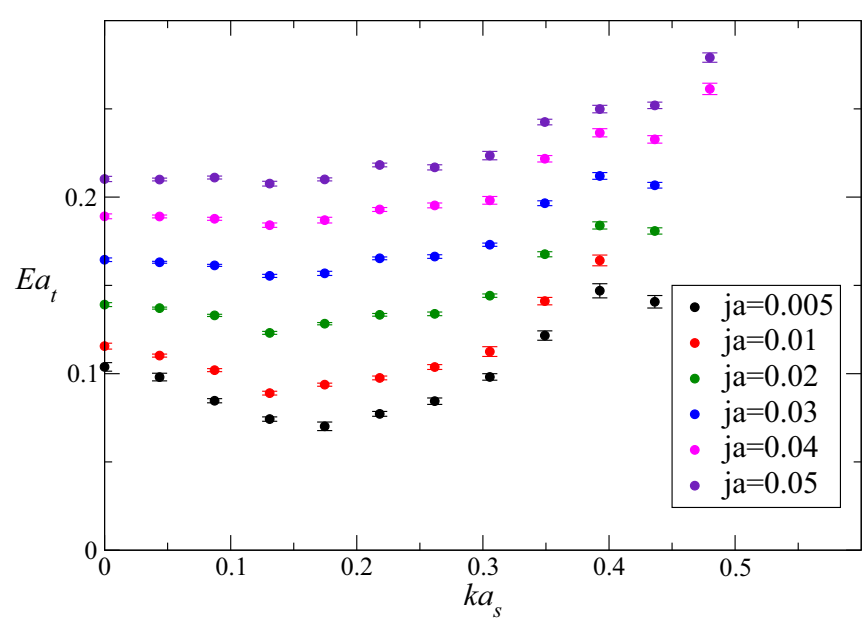

FIG. 3. (Color online) $E(k)$ in the normal channel for $\mu a_{t}=0.1$, for various $j$ on $48^{3}$.

feature is that $E(k)$ is nonmonotonic with a minimum in the neighborhood where the amplitude ratio $A / B \approx 1$, which we have identified as the Fermi momentum $k_{F}$. For $k<k_{F}$ quasiparticle excitations are holelike, and the energy needed to excite them from the ground state decreases as $k \nearrow k_{F}$. For $k>k_{F}$, excitations are particlelike and the opposite holds true: indeed, in this regime results from all three $\mu$ values studied are plausibly consistent with being drawn from the same branch of the dispersion curve appropriate to the vacuum (i.e., with zero bias voltage). Figure 2 compares results from two volumes $32^{3}$ and $48^{3}$, and also for $\mu a_{t}=0.15$ with fits in both normal (14) and anomalous (15) channels. On the assumption that a smooth curve may be drawn through the admittedly noisy data, there is no evidence for any significant finite volume artifacts, or systematic difference between the two channels.

Figure 3 plots $E(k)$ for various $j$ at $\mu a_{t}=0.1$ on $48^{3}$. The nonmonotonicity observed above becomes more pronounced as $j \rightarrow 0$, and since there is little shift in the minimum with $j$, we adopt the pragmatic procedure of identifying the Fermi momentum $k_{F}$ with the value of $k$ where $E$ is minimum. The resulting estimates are shown in Table I; the quoted error is half the mode spacing on $48^{3}$, except at $\mu a_{t}=0.2$, where the dispersion is flatter and a full mode spacing is taken. The first observation is that $k_{F}$ is systematically greater than $\mu$, consistent with the precocious saturation of $n_{c}(\mu)$ observed in [1]. This is discussed further in Sec. IV, but already we note a discrepancy with the expectation $k_{F}=\mu$ for free massless fermions with $a_{s}=a_{t}$. Setting aside the issue of the smearing of the Fermi surface by an exciton gap $\Delta>0$, we can at this stage test consistency with Luttinger's theorem, which

TABLE I. Estimates for the Fermi momentum $k_{F}$ and comparison with Luttinger's theorem.

\begin{tabular}{lclll}
\hline \hline$\mu a_{t}$ & $k_{F} a_{s}$ & $n_{c}^{\text {free }}(\mu) a_{s}^{2}$ & $n_{c}^{\text {free }}\left(k_{F}\right) a_{s}^{2}$ & $n_{c}(\mu, j \rightarrow 0) a_{s}^{2}$ \\
\hline 0.10 & $0.175(22)$ & 0.0032 & $0.011(3)$ & $0.0095(1)$ \\
0.15 & $0.262(22)$ & 0.0080 & $0.023(4)$ & $0.0328(2)$ \\
0.20 & $0.436(44)$ & 0.0139 & $0.068(15)$ & $0.0905(3)$ \\
\hline \hline
\end{tabular}




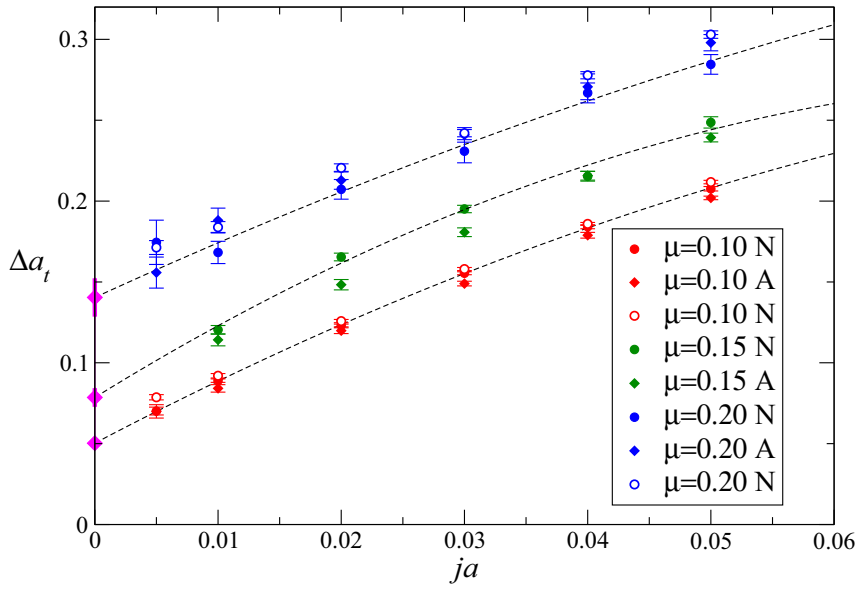

FIG. 4. (Color online) Gap $\Delta$ vs $j$ for various $\mu$. Filled symbols are from $48^{3}$, empty from $32^{3}$, and the dotted lines are fits to normal data on $48^{3}$ using (16).

states that $n_{c}$ depends solely on the geometry of the Fermi surface characterized by $k_{F}$, independent of the nature of the interactions. The third column of Table I shows the carrier density $n_{c}^{\text {free }}$ evaluated for free massless fermions on the same $48^{3}$ lattice at the reference value of $\mu$, and the fourth with $\mu$ set equal to $k_{F}$ in the second column; the fifth column gives the value of $n_{c}$ in the interacting theory in the limit $j \rightarrow 0$ obtained in [1]. In all cases $n_{c}^{\text {free }}(\mu) \ll n_{c}^{\text {free }}\left(k_{F}\right)$, while the values of $n_{c}^{\text {free }}\left(k_{F}\right)$ and $n_{c}(\mu)$ are comparable, which is encouraging; however as $\mu$ increases $n_{c}^{\text {free }}\left(k_{F}\right)<n_{c}(\mu)$ indicative of the difficulties in precisely locating $k_{F}$ due to both the limited momentum resolution and perhaps the absence of a sharp Fermi surface due to exciton condensation.

In Fig. 4 we plot the gap $\Delta$ defined as the energy $E\left(k_{F}\right)$. Data from all available fits on both volumes are shown, in both normal and anomalous channels. As before there is little evidence for a systematic effect with lattice volume and both channels yield consistent results. The dashed lines show an extrapolation to $j=0$ based on the quadratic form

$$
\Delta=\Delta_{0}+a j+b j^{2} .
$$

It is clear $\lim _{j \rightarrow 0} \Delta(j) \neq 0$ which is direct evidence for spontaneous gap formation via exciton condensation for all three values of $\mu$. The resulting extrapolations, together with the estimates for $k_{F}(\mu)$ from Table I are shown in Fig. 5, which is the main result of this paper. In the region of $\mu$ studied it is clear that $\Delta$ varies strongly with and is of the same order of magnitude as the chemical potential $\mu$, which are notable features of this particular model proposed in [1], and indicative of strong interactions at the Fermi surface. This conclusion holds in both normal and anomalous channels, appears to be independent of volume, and is striking enough to be robust against uncertainties introduced by the ad hoc nature of our analysis [an analytic form against which to fit the dispersion $E(k)$ would be very valuable], the IR and UV artifacts inherent in studies of lattice models with $\mu \neq 0$ [16], and lack of knowledge of the physical anisotropy $a_{t} / a_{s}$. On the basis of the three chemical potentials studied both $\Delta$ and $k_{F}$ appear to scale superlinearly with $\mu$, in qualitative agreement with (10).

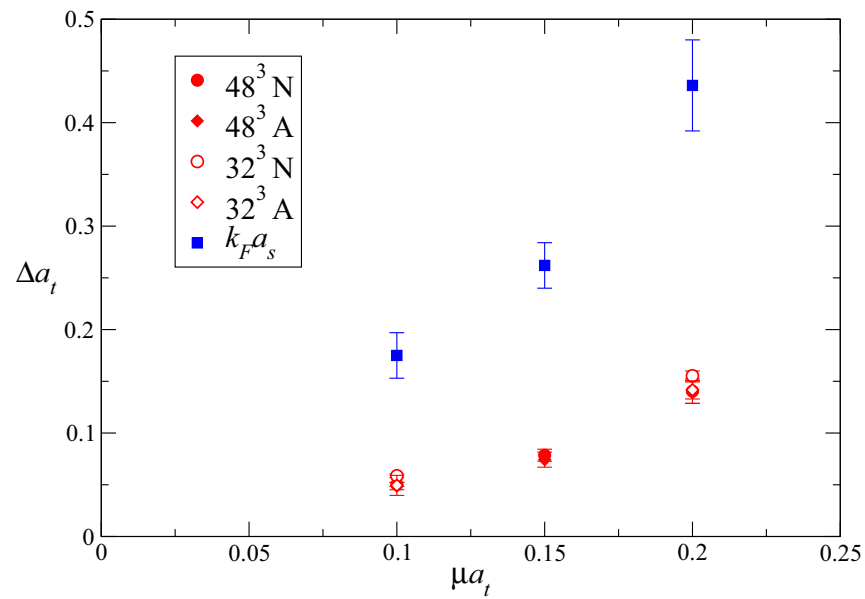

FIG. 5. (Color online) $\Delta(j=0)$ vs $\mu$. The square symbols denote $k_{F}$ from Table I.

\section{DISCUSSION}

In this paper we have used lattice Monte Carlo simulation techniques to explore the quasiparticle dispersion relation in an interacting field theory with nonzero charge density, and shown that for $k \sim k_{F}$ the excitations are gapped with $\Delta \sim O(\mu)$, and $\Delta$ scaling faster than linearly with $\mu$. This is in sharp contrast to results from comparable studies of other simulable models with $\mu \neq 0$. In [13] the gap in the $3+1 d$ Nambu Jona-Lasinio model (a relativistic analog of the original BCS model) was shown to be approximately constant above onset, independent of and numerically much smaller than $\mu$, consistent with the BCS result $\Delta \sim \Lambda_{\mathrm{UV}} \exp \left(-c \Lambda_{\mathrm{UV}}^{2} / \mu^{2}\right)$. In QCD with gauge group $\mathrm{SU}(2)$ there is a so-called quarkyonic regime above onset where $\langle\Psi \Psi\rangle \propto \mu^{2}$ [16]; this is consistent with degenerate fermions in $3+1 d$ with a gap $\Delta \sim O\left(\Lambda_{\mathrm{QCD}}\right)$ independent of $\mu$. It is also very different from the result $\Delta / \mu \sim O\left(10^{-7}\right)$ obtained by self-consistent diagrammatic techniques [7], although comparable with the large values of $\Delta / \mu$ obtained in [5], where it was found that $\Delta$ depends sensitively on the treatment of screening effects, and in particular on the reduction of screening once the superfluid gap forms. One feature of our approach which does merit comparison with the treatment in [5] is that competition between inter- and intralayer pairing condensates can be addressed; see Fig. 11 of [1].

We now return to Table I and the issue of why $\mu a_{t}<k_{F} a_{s}$. In [1] it was suggested this is because in a strongly self-bound system the Fermi energy is necessarily less than the Fermi momentum in natural units. While this may be plausible for a system where $\mu \gg \Delta$ is by far the largest scale, it is difficult to see how this picture can persist in the regime we have been focusing on. Another possibility, which we cannot dismiss, is that the disparity is a lattice artifact caused by a large induced anisotropy $a_{t} / a_{s} \sim O(0.5)$. Indeed, if we assume the Fermi velocity remains close to one even in the presence of interactions, then the dispersion data of Fig. 2 might suggest $a_{t} / a_{s} \approx 0.3$. However, a more compelling possibility is that $E(k)$ is not a linear relation, but rather a power law characteristic of a nearby QCP. Rewrite the scaling form (8) as $n_{c} \propto \mu^{2 / z}$; the Luttinger scaling $n_{c} \propto k_{F}^{2}$ then gives $E \propto k^{z}$, 
where guided by Fig. 2 we assume the relation between $E_{F}$ and $k_{F}$ completely characterizes the quasiparticle dispersion. In this scenario the scaling (8) extracted from bulk observables in [1] thus yields an estimate for the dynamical critical exponent

$$
z \approx 0.6 \text {. }
$$

Much greater numerical precision than achieved in Fig. 2 would be needed to distinguish these two possibilities unambiguously. Another route would be to perform a "biased bilayer" study for a related $2+1 d$ theory, the Thirring model [17], whose behavior as a function of $N_{f}$ and $\mu$ is qualitatively similar to the model here [18], but whose continuum action is manifestly covariant implying $a_{t} \equiv a_{s}$ throughout.

Finally, we note that the superlinear scaling of $\Delta(\mu)$ is also suggestive of a power law $\Delta \propto \mu^{\sigma}$, with $\sigma>1$, modifying our naive expectation $\Delta \propto \mu$. Clearly a much more extensive simulation campaign is required to verify this interesting possibility.

\section{ACKNOWLEDGMENTS}

The authors would like to acknowledge the use of computing resources at Diamond Light Source and the University of Oxford Advanced Research Computing (ARC) facility (http://dx.doi.org/10.5281/zenodo.22558) in carrying out this work. We estimate approximately 1 million core hours of computing time were needed. CPUs used were either Intel Xeon E5-2640v3 Haswell or E5-2650 SandyBridge. S.J.H. was supported by STFC Grant ST/L000369/1, and thanks Allan MacDonald for helpful discussions.
[1] W. Armour, S. Hands, and C. Strouthos, Phys. Rev. D 87, 065010 (2013).

[2] E. V. Castro, K. S. Novoselov, S. V. Morozov, N. M. R. Peres, J. M. B. Lopes dos Santos, J. Nilsson, F. Guinea, A. K. Geim, and A. H. Castro Neto, J. Phys.: Condens. Matter 22, 175503 (2010).

[3] M. Mucha-Kruczyński, I. L. Aleiner, and V. I. Fal'ko, Phys. Rev. B 84, 041404(R) (2011).

[4] J. E. Drut and T. A. Lähde, Phys. Rev. Lett. 102, 026802 (2009); Phys. Rev. B 79, 165425 (2009).

[5] I. Sodemann, D. A. Pesin, and A. H. MacDonald, Phys. Rev. B 85, 195136 (2012).

[6] S. J. Hands and C. G. Strouthos, Phys. Rev. B 78, 165423 (2008).

[7] M. Yu. Kharitonov and K. B. Efetov, Semicond. Sci. Technol. 25, 034004 (2010).

[8] D. V. Khveshchenko, Phys. Rev. Lett. 87, 246802 (2001).
[9] D. T. Son, Phys. Rev. B 75, 235423 (2007).

[10] E. McCann and V. I. Fal'ko, Phys. Rev. Lett. 96, 086805 (2006).

[11] W. Armour, S. Hands, and C. Strouthos, Phys. Rev. B 81, 125105 (2010).

[12] C. J. Burden and A. N. Burkitt, Europhys. Lett. 3, 545 (1987).

[13] S. Hands and D. N. Walters, Phys. Rev. D 69, 076011 (2004).

[14] J. M. Flynn, A. Jüttner, and C. T. Sachrajda, Phys. Lett. B 632, 313 (2006).

[15] S. Hands, B. Lucini, and S. Morrison, Phys. Rev. D 65, 036004 (2002).

[16] S. Cotter, P. Giudice, S. Hands, and J. I. Skullerud, Phys. Rev. D 87, 034507 (2013).

[17] L. Del Debbio, S. Hands, and J. C. Mehegan, Nucl. Phys. B 502, 269 (1997).

[18] S. Christofi, S. Hands, and C. Strouthos, Phys. Rev. D 75, 101701 (2007). 